\title{
Faster Uphill Relaxation in Thermodynamically Equidistant Temperature Quenches
}

\author{
Alessio Lapolla@ and Aljaž Godec®* \\ Mathematical bioPhysics group, Max Planck Institute for Biophysical Chemistry, Göttingen 37077, Germany
}

(Received 6 February 2020; revised 13 June 2020; accepted 10 August 2020; published 11 September 2020)

\begin{abstract}
We uncover an unforeseen asymmetry in relaxation: for a pair of thermodynamically equidistant temperature quenches, one from a lower and the other from a higher temperature, the relaxation at the ambient temperature is faster in the case of the former. We demonstrate this finding on hand of two exactly solvable many-body systems relevant in the context of single-molecule and tracer-particle dynamics. We prove that near stable minima and for all quadratic energy landscapes it is a general phenomenon that also exists in a class of non-Markovian observables probed in single-molecule and particle-tracking experiments. The asymmetry is a general feature of reversible overdamped diffusive systems with smooth singlewell potentials and occurs in multiwell landscapes when quenches disturb predominantly intrawell equilibria. Our findings may be relevant for the optimization of stochastic heat engines.
\end{abstract}

DOI: 10.1103/PhysRevLett.125.110602

Relaxation processes are a paradigm for condensed matter [1,2], single-molecule experiments [3] and tracer-particle transport in complex media [4-8]. Relaxation close to equilibrium was described by the mechanical Onsager-Casimir $[9,10]$ and thermal Kubo-Yokota-Nakajima [11] linear laws. These pioneering ideas were consistently generalized in numerous ways, most notably, to thermodynamics along individual stochastic trajectories driven far from equilibrium at weak $[12,13]$ and strong [14-18] coupling with the bath, anomalous diffusion phenomena [19-22], and the so-called "frenesis" focusing on the dynamical activity-a dynamic counterpart to changes in entropy [23,24]. Many of these new concepts have been verified by and/or successfully applied in experiments in colloidal systems [25-27] and single-molecule experiments on nucleic acids [28-30] and larger biomolecular machines [31].

Not as much is known about transients, in particular those evolving from nonstationary initial conditions. Our present understanding of thermodynamics and in particular the kinetics in transient systems, reversible as well as irreversible, is mostly limited to small deviations from equilibrium $[9,10]$, nonequilibrium steady states [23,32-35], and statistics of the "housekeeping" heat [36,37] and entropy production [38]. The role of initial conditions in relaxation was recently studied in the context of the "Mpemba effect"- the phenomenon where a hot system can cool down faster than the same system initiated at

Published by the American Physical Society under the terms of the Creative Commons Attribution 4.0 International license. Further distribution of this work must maintain attribution to the author(s) and the published article's title, journal citation, and DOI. Open access publication funded by the Max Planck Society. a lower temperature $[39,40]$. Notable recent advances include an information-theoretic bound on the entropy production during relaxation far from equilibrium [41] and a spectral duality between relaxation and first passage processes $[42,43]$.

It is meanwhile possible to probe the transient, nonequilibrium dynamics of colloids and single molecules, e.g., by temperature-modulated particle tracking [4] and timemodulated [44], temperature-modulated [45], temperaturejump [46], and holographic [47] optical tweezers, as well as optical pushing [48]. These experiments allow for systematic investigations of the dependence of relaxation on the direction of the displacement from equilibrium, which is the central question of the present Letter.

Notwithstanding all progress in the field, the dependence of relaxation on the direction of the displacement from
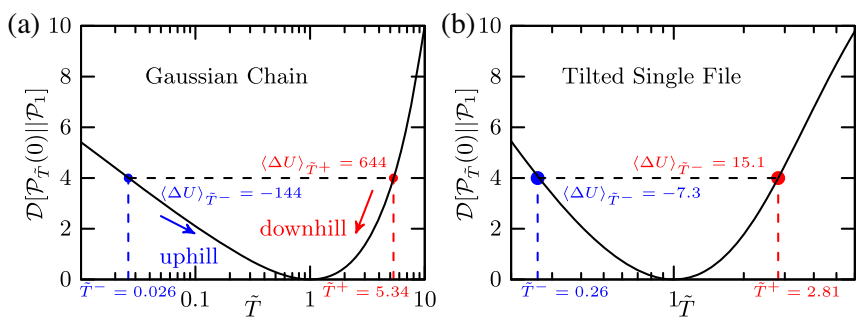

FIG. 1. Nonequilibrium free energy after a temperature quench $T \rightarrow T_{\text {eq }}$ at time $t=0$ in units of $k_{B} T_{\text {eq }}, \Delta F_{\tilde{T}}=\mathcal{D}\left[\mathcal{P}_{\tilde{T}}(t=\right.$ $\left.\left.0^{+}\right) \| \mathcal{P}_{1}^{\mathrm{eq}}\right]$ [see Eq. (3)], as a function of the relative prequench temperature $\tilde{T}=T / T_{\text {eq }}$ (note the logarithmic scale); (a) refers to the end-to-end distance of a Gaussian chain with 100 beads and (b) to the 7 th in a single file of 10 particles in a linear potential with slope 10 confined to a unit box. The blue and red points depict a pair of thermodynamically equidistant temperature quenches, $\tilde{T}^{-}$and $\tilde{T}^{+}$, with corresponding excess potential energies $\langle\Delta U\rangle_{\tilde{T}^{ \pm}} \equiv\left\langle U\left(0^{+}\right)\right\rangle_{\tilde{T}^{ \pm}}-\langle U\rangle_{1}$. 
equilibrium (see Fig. 1) remains elusive. Moreover, as a result of the projection to a lower-dimensional subspace, it is expected that observables in many experiments, in particular those tracking individual particles [4] and single molecules [46,47], relax in a manner that is not Markovian [8].

Here, we address relaxation from an instantaneous temperature quench $T \rightarrow T_{\text {eq }}$ at time $t=0$ with respect to its directionality, that is, $T^{-\uparrow} T_{\text {eq }}$ versus $T^{+} \downarrow T_{\text {eq }}$. We uncover an unforeseen dependence on the direction of the quench: For a given pair of temperatures $T^{-}<T_{\text {eq }}<T^{+}$at which the thermodynamic displacement from equilibrium at $t=0^{+}$in the sense of $\mathcal{D}_{T^{ \pm}}\left(0^{+}\right)$, the nonequilibrium free energy difference or "lag" [49-55], is equal, i.e., $\mathcal{D}_{T^{+}}\left(0^{+}\right)=\mathcal{D}_{T^{-}}\left(0^{+}\right)$(see Fig. 1), relaxation evolves, contrary to intuition, faster "uphill" $\left(\langle\Delta U\rangle_{T^{-}}<0\right)$ than "downhill" $\left(\langle\Delta U\rangle_{T^{+}}>0\right)$ in the energy landscape. This always holds for single-well potentials and occurs in near degenerate multiwell potentials with high energy barriers under Markovian dynamics, as well as for a class of non-Markovian observables probed by single-molecule and particle-tracking experiments. We demonstrate the asymmetry on the hand of the Gaussian polymer chain [56], single-file diffusion in a tilted box [8], and for diffusion in nearly degenerate multiwell potentials. For relaxation near a stable minimum and thus for all reversible OrnsteinUhlenbeck processes, we prove that the asymmetry, albeit counterintuitive, is general.

Theory.-We consider $d$-dimensional Markovian diffusion with a $d \times d$ symmetric positive-definite diffusion matrix $\mathbf{D}$ and mobility tensor $\mathbf{M}_{T}=\mathbf{D} / k_{B} T$ in a drift field $\mathbf{F}(\mathbf{x})$ such that $\mathbf{M}_{T}^{-1} \mathbf{F}(\mathbf{x})=-\nabla U(\mathbf{x})$ is a gradient flow. The evolution of the probability density at temperature $T$ is governed by the Fokker-Planck operator $\hat{\mathcal{L}}_{T} \equiv \nabla \cdot \mathbf{D} \nabla-\nabla \cdot \mathbf{M}_{T} \mathbf{F}(\mathbf{x})$. We let $G_{T}\left(\mathbf{x}, t \mid \mathbf{x}_{0}\right)$ be the Green's function of the initial value problem $\left(\partial_{t}-\right.$ $\left.\hat{\mathcal{L}}_{T}\right) G_{T}\left(\mathbf{x}, t \mid \mathbf{x}_{0}\right)=0$ and assume that the potential $U(\mathbf{x})$ is confining (i.e., $\lim _{|\mathbf{x}| \rightarrow \infty} U(\mathbf{x})=\infty$ ). This assures the existence of an invariant Maxwell-Boltzmann measure with density $\lim _{t \rightarrow \infty} G_{T}\left(\mathbf{x}, t \mid \mathbf{x}_{0}\right) \equiv P_{T}^{\mathrm{eq}}(\mathbf{x})=$ $Q_{T}^{-1} \mathrm{e}^{-U(\mathbf{x}) / k_{B} T}, \quad \forall \mathbf{x}_{0}$ with partition function $Q_{T}=$ $\int \mathrm{e}^{-U(\mathbf{x}) / k_{B} T} d \mathbf{x}$.

The system is prepared at equilibrium with a temperature $T, P_{T}^{\text {inv }}(\mathbf{x})$, whereupon an instantaneous temperature quench is performed to the ambient temperature $T_{\text {eq }}$ at $t=0$. The relaxation evolves at $T_{\text {eq }}$ according to $\hat{\mathcal{L}}_{T_{\mathrm{eq}}}$ and for a given system it is uniquely characterized by $T$. For convenience we define $\tilde{T} \equiv T / T_{\text {eq }}[57]$ such that

$$
P_{\tilde{T}}(\mathbf{x}, t)=\int d \mathbf{x}_{0} G_{1}\left(\mathbf{x}, t \mid \mathbf{x}_{0}\right) P_{\tilde{T}}^{\mathrm{eq}}\left(\mathbf{x}_{0}\right) \underset{t \rightarrow \infty}{\rightarrow} P_{1}^{\mathrm{eq}}(\mathbf{x}) .
$$

The instantaneous entropy and mean energy are given by $S_{\tilde{T}}(t) \equiv-k_{B} \int d \mathbf{x} P_{\tilde{T}}(\mathbf{x}, t) \ln P_{\tilde{T}}(\mathbf{x}, t) \quad$ and $\langle U(t)\rangle_{\tilde{T}}=\int d \mathbf{x} P_{\tilde{T}}(\mathbf{x}, t) U(\mathbf{x})$, respectively, where $\langle\cdot\rangle_{\tilde{T}}$ denotes an average over all paths $\mathbf{x}(t)$ starting from $P_{\tilde{T}}^{\text {inv }}\left(\mathbf{x}_{0}\right)$.

Let the measured physical observable be $\mathbf{q}=\boldsymbol{\Gamma}(\mathbf{x})$. Its probability density function corresponds to [8]

$\mathcal{P}_{\tilde{T}}(\mathbf{q}, t)=\hat{\Pi}_{\mathbf{x}}(\mathbf{q}) P_{\tilde{T}}(\mathbf{x}, t) \equiv \int d \mathbf{x} \delta[\boldsymbol{\Gamma}(\mathbf{x})-\mathbf{q}] P_{\tilde{T}}(\mathbf{x}, t),(2)$

which in general displays non-Markovian dynamics as soon as $\mathbf{q}$ corresponds to a low-dimensional projection [8]. Once equilibrium is reached, we have $\lim _{t \rightarrow \infty} \mathcal{P}_{\tilde{T}}(\mathbf{q}, t)=\mathcal{P}_{1}^{\mathrm{eq}}(\mathbf{q})$, or, expressed via the so-called potential of mean force $\mathcal{U}(\mathbf{q})$ [58], $\mathcal{P}_{1}^{\text {eq }}(\mathbf{q})=\mathrm{e}^{-\beta_{\text {eq }} \mathcal{U}(\mathbf{q})}$ [14,17,59]. Obviously, when $\boldsymbol{\Gamma}(\mathbf{x})=\mathbf{x}$, we have $\mathcal{P}_{\tilde{T}}(\mathbf{q}, t)=P_{\tilde{T}}(\mathbf{x}, t)$.

We quantify the instantaneous displacement from equilibrium with the Kullback-Leibler divergence [49-55]:

$\mathcal{D}\left[\mathcal{P}_{\tilde{T}}(t) \| \mathcal{P}_{1}^{\mathrm{eq}}\right]=\int d \mathbf{q} \mathcal{P}_{\tilde{T}}(\mathbf{q}, t) \ln \left[\mathcal{P}_{\tilde{T}}(\mathbf{q}, t) / \mathcal{P}_{1}^{\mathrm{eq}}(\mathbf{q})\right]$.

Writing this out for the Markovian case, we find, upon identifying $S_{\tilde{T}}(t)$ and $\left\langle U_{\tilde{T}}(t)\right\rangle$,

$\mathcal{D}\left[P_{\tilde{T}}(t) \| P_{1}^{\mathrm{eq}}\right]=-S_{\tilde{T}}(t) / k_{B}+\beta_{\mathrm{eq}}\langle U(t)\rangle_{\tilde{T}}+\ln Q_{T_{\mathrm{eq}}}$.

Recalling the definition of free energy $F=-\beta_{\mathrm{eq}}^{-1} \ln Q_{T_{\mathrm{eq}}}$ and defining the instantaneous generalized free energy (GFE) [52] or "lag" [55] as $F_{\tilde{T}}(t)=\langle U(t)\rangle_{\tilde{T}}-T_{\text {eq }} S_{\tilde{T}}(t)$, we see, upon multiplying through by $\beta_{\text {eq }}^{-1}=k_{B} T_{\text {eq }}$, that in the Markovian case Eq. (3) is the excess GFE in units of $k_{B} T_{\text {eq }}$, i.e., $\mathcal{D}_{\tilde{T}}^{M}(t) \equiv \mathcal{D}\left[P_{\tilde{T}}(t) \| P_{1}^{\mathrm{eq}}\right]=\beta_{\text {eq }}\left[F_{\tilde{T}}(t)-F\right]$ [51,52]. Writing out Eq. (3) for the non-Markovian case and identifying $\mathcal{S}_{\tilde{T}}(t)$ and $\mathcal{U}(\mathbf{q})$ (calligraphic letters denote potentials of projected observables), we find

$\mathcal{D}_{\tilde{T}}^{n M}(t) \equiv \mathcal{D}\left[\mathcal{P}_{\tilde{T}}(t) \| \mathcal{P}_{1}^{\mathrm{eq}}\right]=-\mathcal{S}_{\tilde{T}}(t) / k_{B}+\beta_{\mathrm{eq}}\langle\mathcal{U}(t)\rangle_{\tilde{T}}$,

which is the non-Markovian GFE $\mathcal{D}_{\tilde{T}}^{n M}(t)=\beta_{\text {eq }} \mathcal{F}_{\tilde{T}}(t)$. Note that $\mathcal{U}(\mathbf{q})$ itself is an effective free energy, i.e., $\quad \beta_{\text {eq }} \mathcal{U}(\mathbf{q}) \equiv-\ln \langle\delta[\boldsymbol{\Gamma}(\mathbf{x})-\mathbf{q}]\rangle_{1}=-\ln \int d \mathbf{x} \delta[\boldsymbol{\Gamma}(\mathbf{x})-$ $\mathbf{q}] \mathrm{e}^{-\beta_{\mathrm{eq}} U(\mathbf{x})}+\ln Q_{T_{\mathrm{eq}}}$ and $\mathcal{S}_{1}=-\langle\mathcal{U}\rangle_{1}$. We henceforth express energies in units of $k_{B} T_{\text {eq. }}$. If (and only if) latent degrees of freedom (i.e., those integrated out) relax much faster than $\mathbf{q}(t)$, Eqs. (4) and (5) are equivalent and $\mathbf{q}(t)$ is a Markovian diffusion in the free energy landscape $\mathcal{U}(\mathbf{q})$ [8]. In the absence of a time-scale separation, however, both $\mathcal{S}_{\tilde{T}}(t)$ and $\langle\mathcal{U}(t)\rangle_{\tilde{T}}$ contain contributions from the (hidden) relaxation of the latent degrees of freedom.

Consider now a pair of temperatures, $\tilde{T}^{+}>1$ and $\tilde{T}^{-}<1$, corresponding to equal displacements immediately after the quench $\mathcal{D}_{\tilde{T}^{-}}^{M, n M}\left(0^{+}\right)=\mathcal{D}_{\tilde{T}^{+}}^{M, n M}\left(0^{+}\right)$. The existence of (at least) two such temperatures is guaranteed within an interval $\tilde{T} \in\left(\tilde{T}_{\min }, \tilde{T}_{\max }\right)$ where $\mathcal{D}_{\tilde{T}}^{M, n M}\left(0^{-}\right)=f(\tilde{T})$ has 

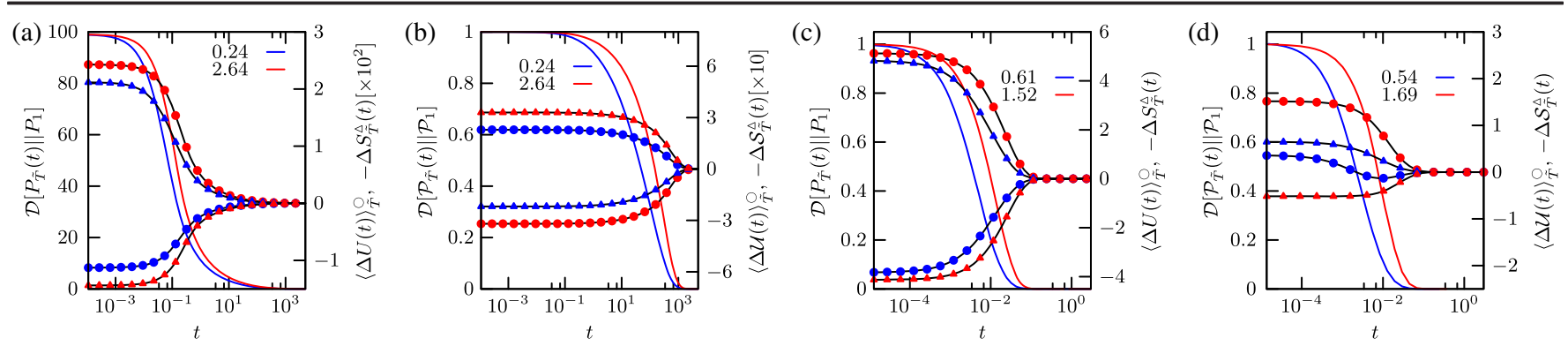

FIG. 2. $\mathcal{D}\left[\mathcal{P}_{\tilde{T}}(t) \| \mathcal{P}_{1}^{\text {eq }}\right]$ (full lines) for the Gaussian chain [(a),(b)] and single file with 10 particles in a linear potential with slope $g=10$ [(s),(d)]. (a) refers to the entire chain of 100 beads [Eq. (4)] and (b) to the end-to-end distance [Eq. (5)] for equidistant quenches from $\tilde{T}^{-}=0.24$ (blue) and $\tilde{T}^{+}=2.64$ (red); (c) stands for the full single file for equidistant quenches from $\tilde{T}^{-}=0.61$ (blue) and $\tilde{T}^{+}=1.52$ (red); (d) the 7th particle for equidistant quenches from $\tilde{T}^{-}=0.54$ (blue) and $\tilde{T}^{+}=1.69$. The circles refer to $\langle\Delta U(t)\rangle_{\tilde{T}^{ \pm}}$and $\langle\Delta \mathcal{U}(t)\rangle_{\tilde{T}^{ \pm}}$ in (a) and (c), and (b) and (d), respectively, and triangles denote $\Delta S_{\tilde{T}^{ \pm}}(t)$ and $\Delta \mathcal{S}_{\tilde{T}}(t)$. Note the second axes for $\langle\Delta U(t)\rangle_{\tilde{T}^{ \pm}},\langle\Delta \mathcal{U}(t)\rangle_{\tilde{T}^{ \pm}}$ and $\Delta S_{\tilde{T}^{ \pm}}(t), \Delta \mathcal{S}_{\tilde{T}}(t)$. Note that $\mathcal{S}_{\tilde{T}}(\infty)=\mathcal{S}_{1}=-\langle\mathcal{U}\rangle_{1}$.

no local maximum. The central question of this Letter addresses the rate of the "uphill" $\left(\tilde{T}^{-}<1\right)$ versus "downhill" $\left(\tilde{T}^{+}>1\right)$ relaxation.

Gaussian Chain.-In the context of single-molecule experiments, we consider the overdamped dynamics of a chain of $N+1$ beads with coordinates $\left\{\mathbf{r}_{i}\right\}$ connected by harmonic springs with potential $U\left(\left\{\mathbf{r}_{i}\right\}\right)=$ $\sum_{i=1}^{N}\left(\mathbf{r}_{i+1}-\mathbf{r}_{i}\right)^{2}$ (general harmonic networks are treated in [60]). In the Markovian setting, we consider all monomers, $P_{\tilde{T}}\left(\left\{\mathbf{r}_{i}\right\}, t\right)$ in Eq. (1), while single-molecule experiments (e.g., Förster resonance energy transfer $[73,74]$ or optical tweezers $[46,47])$ typically track a single (e.g., end to end) distance within the macromolecule $\mathbf{q} \equiv d=$ $\left|\mathbf{r}_{1}-\mathbf{r}_{N}\right|$ with $\mathcal{P}_{\tilde{T}}(d, t)$ from Eq. (2), evolving according to non-Markovian dynamics.

The excess GFE is given by (see derivation in [60])

$$
\begin{gathered}
\mathcal{D}_{\tilde{T}}^{M}(t)=\frac{3}{2} \sum_{k=1}^{N}\left[\Lambda_{k}^{\tilde{T}}(t)-1-\ln \Lambda_{k}^{\tilde{T}}(t)\right] \\
\mathcal{D}_{\tilde{T}}^{n M}(t)=\frac{3}{2}\left[\frac{\mathcal{A}_{\tilde{T}}^{1 N}(t)}{\mathcal{A}_{1}^{1 N}(0)}-1-\ln \frac{\mathcal{A}_{\tilde{\tilde{T}}}^{1 N}(t)}{\mathcal{A}_{1}^{1 N}(0)}\right],
\end{gathered}
$$

where $\quad \Lambda_{k}^{\tilde{T}}(t) \equiv 1+(\tilde{T}-1) \mathrm{e}^{-2 \mu_{k} t} \quad$ with $\quad \mu_{k}=$ $4 \sin ^{2}\{k \pi /[2(N+1)]\}$, and we introduced $\mathcal{A}_{\tilde{T}}^{i j}(t) \equiv$ $\sum_{k=1}^{N} \Lambda_{k}^{\tilde{T}}(t) \mathcal{C}_{k}^{i j} / 2 \mu_{k}$ with $\mathcal{C}_{k}^{i j} \geq 0$ given explicitly in [60]. The initial excess free energies are both convex in $\tilde{T}$ and read

$$
\mathcal{D}_{\tilde{T}}^{M}\left(0^{+}\right)=3 N(\tilde{T}-1-\ln \tilde{T}) / 2=N \mathcal{D}_{\tilde{T}}^{n M}\left(0^{+}\right) .
$$

The instantaneous potential energy of the full system and the potential of mean force in turn read $\langle U(t)\rangle_{\tilde{T}}=$ $\frac{3}{2} \sum_{k=1}^{N} \Lambda_{k}^{\tilde{T}}(t) \quad$ and $\quad \mathcal{U}(d)=-\ln \mathcal{P}_{1}^{\mathrm{eq}}(d), \quad$ respectively. Aside from specific values of $\mu_{k}$ and $\mathcal{C}_{k}^{i j}$, Eqs. (6)-(8) hold for any reversible Ornstein-Uhlenbeck" process (OUp), that is, for any $\tilde{T}$, connectivity or stability matrix, and tagged distance.

The results for $\mathcal{D}_{\tilde{T}}^{M, n M}(t)$ and their decomposition into $\langle U\rangle_{\tilde{T}},\langle\mathcal{U}(t)\rangle_{\tilde{T}}, S_{\tilde{T}}(t)$, and $\mathcal{S}_{\tilde{T}}(t)$ for a pair of equidistant temperature quenches are shown in Fig. 2 and demonstrate that the uphill relaxation is always faster than the downhill relaxation. As we prove below, this is true for any reversible OUp quenched arbitrarily far from equilibrium.

The energy and entropy differences relative to their equilibrium values (i.e., at $t=\infty$ ) in Fig. 2(a) suggest that the Markovian uphill and downhill relaxation are dominated by $\langle\Delta U(t)\rangle_{\tilde{T}^{+}}$and $\Delta S_{\tilde{T}^{-}}$, respectively. Surprisingly, entropy pushing the system uphill against the deterministic force is more efficient. Notably, the magnitude of individual contributions is smaller for uphill relaxation, i.e., $\langle\Delta U\rangle_{\tilde{T}^{+}}>-\langle\Delta U\rangle_{\tilde{T}^{-}}$and $\Delta S_{\tilde{T}^{+}}>-\Delta S_{\tilde{T}^{-}}$. Thus, a larger energy excess and entropy deficit are dissipated during downhill relaxation. Conversely, the partitioning into $\mathcal{S}_{\tilde{T}}(t)$ and $\langle\mathcal{U}(t)\rangle_{\tilde{T}}$ of the non-Markovian relaxation depends on the details of the projection and is less intuitive [in our example in Fig. 2(b) it is, in fact, reversed].

To explain why uphill relaxation is faster, we inspect in Fig. 3 local contributions to $\mathcal{D}_{\widetilde{T}}^{M}(t)$ for a one-dimensional OUp. An uphill quench localizes $P_{\tilde{T}^{-}}\left(x, 0^{+}\right)$near the origin, whereas a downhill quench broadens $P_{\tilde{T}^{+}}\left(x, 0^{+}\right)$, rendering the integrand of Eq. (4) nonzero over a larger domain [Fig. 3(a), red line]. The evolution of $P_{\tilde{T}}(x, t)$ is driven by diffusion $\propto \partial_{x}^{2} P_{\tilde{T}}$ and advection $\propto \partial_{x} x P_{\tilde{T}}$. By forcing probability mass toward the origin, advection seems to oppose uphill relaxation [triangles in Fig. 3(b)] but thereby actually sustains an even faster diffusion rate compared to free diffusion [compare circles and dashed line in Fig. 3(b)]. The net effect is an overall relaxation nearly as fast as free diffusion [compare full and dashed line in Fig. 3(b)]. Downhill relaxation is advection-dominated and weakly opposed by diffusion, which is almost unaffected by the potential [Fig. 3(c)]. The overall dynamics is much slower [compare full lines in Fig. 3(b), (c)]. Faster diffusion from a localized initial distribution thereby renders uphill 


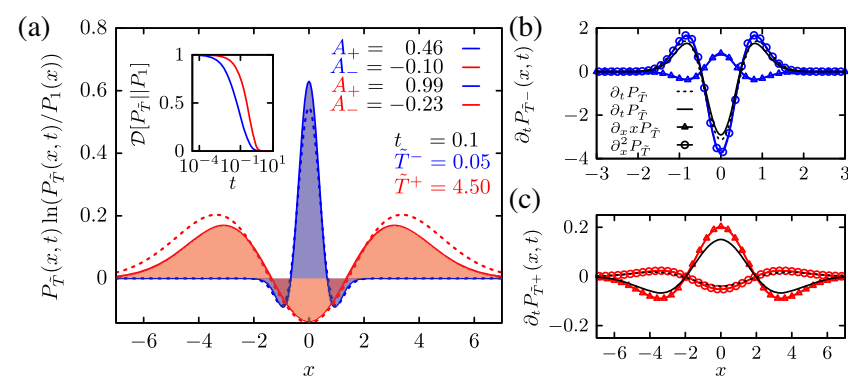

FIG. 3. (a) Integrand of Eq. (3) at $t=0.1$ for a one-dimensional OUp (full line) for uphill (blue) and downhill (red) relaxation with the positive $A_{+}$and negative $A_{-}$area under the curve. Inset: The corresponding $\mathcal{D}_{\tilde{T}}^{M}(t)$. (b), (c) Decomposition of $\partial_{t} P_{\tilde{T}}(x, t)$ into diffusive $\partial_{x}^{2} P_{\tilde{T}}$ (circles) and advective $\partial_{x} x P_{\tilde{T}}$ (triangles) contribution for uphill (b) and downhill (c) relaxation. Dashed lines correspond to free diffusion evolving from the same initial condition.

relaxation faster - an effect that will exist in any confining potential well with ruggedness $\ll k_{B} T_{\text {eq }} . \hat{\mathcal{L}}_{T}$ of any reversible OUp is diagonalizable and thus uniquely decomposable into one-dimensional OUps, extending our explanation to arbitrary dimensions.

Non-Markovian relaxation displays the same asymmetry, but the dominant driving forces, here $\mathcal{S}_{\tilde{T}}$ and $\left\langle\mathcal{U}_{\tilde{T}}\right\rangle$, may become reversed [see Fig. 2(b)]. Since $\mathcal{U}_{\tilde{T}}$ contains entropic effects of latent degrees of freedom, the partitioning between $\mathcal{S}_{\tilde{T}}$ and $\left\langle\mathcal{U}_{\tilde{T}}\right\rangle$ is in general projectiondependent.

Tilted single file.-In the context of tracer-particle dynamics, we consider $N$ hard-core Brownian point particles with positions $\left\{x_{i}(t)\right\}$ (the extension to a finite diameter is straightforward [5,6]) diffusing in a box of unit length in the presence of a linear potential (e.g., the gravitational field), $U\left(\left\{x_{i}\right\}\right)=\sum_{i=1}^{N} g x_{i}$. The probability density of $\left\{x_{i}(t)\right\}$ upon a quench from $\tilde{T}, P_{\tilde{T}}\left(\left\{x_{i}(t)\right\}, t\right)$, evolves according to $\hat{\mathcal{L}}_{1}=\sum_{i=1}^{N}\left(\partial_{x_{i}}^{2}+g \partial_{x_{i}}\right)$ under noncrossing conditions $[7,8]$. In [60] we solve the problem exactly via the coordinate Bethe ansatz $[7,8]$, both for the Markovian complete single file and the nonMarkovian probability density of a tagged particle $\mathcal{P}_{\tilde{T}}(z, t)$ (i.e., $\mathbf{q} \equiv x_{\mathcal{T}}=z$ ).

$\mathcal{D}_{\tilde{T}}^{M}(t)$ with corresponding $\langle\Delta U(t)\rangle_{\tilde{T}},\langle\mathcal{U}(t)\rangle_{\tilde{T}}, \Delta S_{\tilde{T}}(t)$, and $\mathcal{S}_{\tilde{T}}(t)$ for the complete and tagged particle dynamics are shown in Fig. 2(c), (d). As for the Gaussian chain uphill relaxation in the tilted single file, both full as well as for a tagged particle seems to always be faster irrespective of which particle we tag, and for any $\tilde{T}, N$ and tilting strength $g>0$ (see also [60]). The Markovian uphill relaxation is dominated by $\Delta S_{\tilde{T}^{-}}(t)$ and downhill by $\langle\Delta U(t)\rangle_{\tilde{T}^{+}}$, and a larger energy and entropy difference must be dissipated during downhill relaxation [see Fig. 2(c)]. For a tagged particle, the partitioning between $\langle\mathcal{U}(t)\rangle_{\tilde{T}^{-}}$and $\mathcal{S}(t)_{\tilde{T}^{-}}$ varies depending on which particle we tag as a result of the shape of $\mathcal{U}(z)$ and the dependence of $\mathcal{P}_{\tilde{T}}\left(z, 0^{-}\right)$on $\tilde{T}$, which in turn both depend on the tagged particle as well as $T_{\text {eq }}, N$, and $g$.

Is the asymmetry universal?-We first focus on dynamics near a stable minimum at $\mathbf{R}_{0}, \delta \mathbf{R}(t)=\mathbf{R}(t)-\mathbf{R}_{0}$, which is well described by an OUp, i.e., $d \delta \mathbf{R}(t)=$ $\mathbf{H} \delta \mathbf{R}(t) d t+\sqrt{2} d \mathbf{W}_{t}$, where $(\mathbf{H})_{i j}=\left.\sum_{i j} \partial_{R_{i}} \partial_{R_{j}} U(\mathbf{R})\right|_{\mathbf{R}_{0}}$ is the Hessian.

Theorem 1.-For a general diffusion sufficiently close to a stable minimum and for any stable reversible OUp, the relaxation from a pair of equidistant quenches of arbitrary magnitude (as defined above) is always faster uphill.

Proof of Theorem 1.-Any pair $0<\tilde{T}^{-} \leq 1$ and $1 \leq$ $\tilde{T}^{+}<\infty$ with $\mathcal{D}_{\tilde{T}^{+}}\left(0^{+}\right)=\mathcal{D}_{\tilde{T}^{-}}\left(0^{+}\right)$satisfies by construction $\tilde{T}^{+}-\tilde{T}^{-}=\ln \left(\tilde{T}^{+} / \tilde{T}^{-}\right)$. We first prove the claim for the Markovian setting, where Eq. (6) has the structure $\mathcal{D}_{\tilde{T}}^{M}(t)=\sum_{k=1}^{N} \mathcal{D}_{k}^{ \pm}(t)$. We set $\varphi \equiv \tilde{T}^{+} / \tilde{T}^{-}>1$, $\delta_{ \pm} \equiv \tilde{T}^{ \pm}-1$, and write $\Delta \mathcal{D}_{k}(t) \equiv \mathcal{D}_{k}^{+}(t)-\mathcal{D}_{k}^{-}(t)=$ $\ln Z_{\varphi}\left(\mu_{k} t\right)$, such that

$$
\begin{aligned}
Z_{\varphi}(\tau) & =\varphi^{\mathrm{e}^{-\tau}}\left(1+\delta_{-} \mathrm{e}^{-\tau}\right) /\left(1+\delta_{+} \mathrm{e}^{-\tau}\right) \\
& =\left[\varphi\left(1+\delta_{-} \mathrm{e}^{-\tau}\right)^{\mathrm{e}^{\tau}}\right] \mathrm{e}^{-\tau} /\left(1+\delta_{+} \mathrm{e}^{-\tau}\right) \\
& \geq\left[\varphi\left(1+\delta_{-}\right) /\left(1+\delta_{+}\right)\right]^{\mathrm{e}^{-\tau}} \geq 1,
\end{aligned}
$$

where we have used both generalized Bernoulli inequalities, i.e., for any real $0 \leq y_{-} \leq 1, y_{+} \geq 1$ and $x \geq-1$ we have $(1+x)^{y_{+}} \geq 1+y_{+} x$ and $(1+x)^{y_{-}} \leq 1+y_{-} x$. Recalling the definition of $\Delta \mathcal{D}_{k}(t)$ completes the proof.

To prove the claim in the non-Markovian setting for projections of type $q=\left|\delta \mathbf{R}_{i}-\delta \mathbf{R}_{j}\right|$, we first realize that $\dot{\mathcal{A}}_{\widetilde{T}^{+}}^{i j}(t) \leq 0$ and $\dot{\mathcal{A}}_{\tilde{T}^{-}}^{i j}(t) \geq 0$, where $\dot{f}(t) \equiv(d / d t) f(t)$. Setting $\Delta \mathcal{D}(t) \equiv \mathcal{D}_{\tilde{T}^{+}}^{n M}(t)-\mathcal{D}_{\tilde{T}^{-}}^{n M}(t)$ and using Eq. (7) we find, upon taking the derivative,

$$
\Delta \dot{\mathcal{D}}(t)=\frac{\dot{\mathcal{A}}_{\tilde{T}^{+}}^{i j}(t)}{\mathcal{A}_{\tilde{T}^{+}}^{i j}(0)}-\frac{\dot{\mathcal{A}}_{\tilde{T}^{+}}^{i j}(t)}{\mathcal{A}_{\tilde{T}^{+}}^{i j}(t)}-\frac{\dot{\mathcal{A}}_{\tilde{T}^{-}}^{i j}(t)}{\mathcal{A}_{\tilde{T}^{-}}^{i j}(0)}+\frac{\dot{\mathcal{A}}_{\tilde{T}^{-}}^{i j}(t)}{\mathcal{A}_{\tilde{T}^{-}}^{i j}(t)}
$$

Eq. (10) implies $\Delta \dot{\mathcal{D}}(t) \geq 0$ because $\mathcal{A}_{\tilde{T}^{+}}^{i j}(t) \geq \mathcal{A}_{\tilde{T}^{+}}^{i j}(0)$ while $\mathcal{A}_{\tilde{T}^{-}}^{i j}(t) \leq \mathcal{A}_{\tilde{T}^{+}}^{i j}(0)$, which completes the proof.

The fact that tilted single file diffusion, being anharmonic and asymmetric with nonperturbative interactions, displays the asymmetry for quenches of arbitrary magnitude and for any steepness of the potential hints that the asymmetry might be more general. Note that tagging different particles in different slopes $g>0$, we can construct $\mathcal{U}(z)$ with arbitrary asymmetry. Alongside the physical principle underlying the asymmetry established for the OUp and Theorem 1, this strongly suggests that uphill relaxation in smooth single-well potentials could be universally faster (see [60]). Since the projection (2) is independent of $\tilde{T}$, these statements should extend also to non-Markovian observables, in particular those probed in many single-molecule and particle-tracking experiments. 
As a corollary, uphill relaxation is faster also in multiwell potentials for equidistant quenches that predominantly disturb only the intrawell equilibria, in particular for nearly degenerate basins separated by sufficiently high barriers [75] (for reasoning and examples, see [60]). This is violated in asymmetric multiwells, and examples with faster downhill relaxation are constructed in [60].

Conclusion.-We uncovered an unforeseen asymmetry in the relaxation to equilibrium in equidistant temperature quenches. Uphill relaxation was found to be faster-a phenomenon we proved to be universal for quenches of dynamics near stable minima. We hypothesize that it is a general phenomenon in reversible overdamped diffusion in single-well potentials extending to degenerate multiwell potentials for quenches leaving interwell equilibria virtually intact. The dependence on the direction of the quench, which so far seems to have been overlooked, implies a systematic asymmetry in the dissipation of the system's entropy $\dot{S}_{\tilde{T}}(t)$ versus heat $\langle\dot{U}(t)\rangle_{\tilde{T}}[12]$ and, for specific projections, the modified entropy $\dot{\mathcal{S}}_{\tilde{T}}(t)$ versus "strong coupling heat" $\langle\dot{\mathcal{U}}(t)\rangle_{\tilde{T}}[14,17]$, which seems to be relevant for the efficiency of stochastic heat engines [44,76,77]. Implying that the hot isothermal step can be shorter than the cold one, which reduces cycle times, the asymmetry may also be relevant for the optimization of the engine's output power [44,76,77]. Our results can readily be tested by single-molecule and particle-tracking experiments [4,44-48]. To understand the asymmetry on the level of individual trajectories, it would be interesting to analyze relaxation from equidistant quenches in terms of occupation measures $[7,8]$ and from the perspective of stochastic thermodynamics $[12,14]$.

We thank David Hartich for fruitful discussions. The financial support from the German Research Foundation (DFG) through the Emmy Noether Program GO 2762/1-1 to A. G. is gratefully acknowledged.

*Corresponding author. agodec@mpibpc.mpg.de

[1] A. Farhan, P. M. Derlet, A. Kleibert, A. Balan, R. V. Chopdekar, M. Wyss, J. Perron, A. Scholl, F. Nolting, and L. J. Heyderman, Direct Observation of Thermal Relaxation in Artificial Spin Ice, Phys. Rev. Lett. 111, 057204 (2013).

[2] S. Dattagupta, Relaxation Phenomena in Condensed Matter Physics (Elsevier, New York, 2012).

[3] H. Chen, E. Rhoades, J. S. Butler, S. N. Loh, and W. W. Webb, Dynamics of equilibrium structural fluctuations of apomyoglobin measured by fluorescence correlation spectroscopy, Proc. Natl. Acad. Sci. U.S. A. 104, 10459 (2007).

[4] P. Wang, C. Song, and H. A. Makse, Dynamic particle tracking reveals the ageing temperature of a colloidal glass, Nat. Phys. 2, 526 (2006).

[5] L. Lizana and T. Ambjörnsson, Single-File Diffusion in a Box, Phys. Rev. Lett. 100, 200601 (2008).
[6] L. Lizana and T. Ambjörnsson, Diffusion of finite-sized hard-core interacting particles in a one-dimensional box: \{Tagged\} particle dynamics, Phys. Rev. E 80, 051103 (2009).

[7] A. Lapolla and A. Godec, Unfolding tagged particle histories in single-file diffusion: exact single- and two-tag local times beyond large deviation theory, New J. Phys. 20, 113021 (2018).

[8] A. Lapolla and A. Godec, Manifestations of projectioninduced memory, Front. Phys. 7, 182 (2019).

[9] L. Onsager, Reciprocal relations in irreversible processes I, Phys. Rev. 37, 405 (1931).

[10] L. Onsager, Reciprocal relations in irreversible processes II, Phys. Rev. 38, 2265 (1931).

[11] R. Kubo, M. Yokota, and S. Nakajima, Statistical-Mechanical Theory of Irreversible Processes. II. Response to Thermal Disturbance, J. Phys. Soc. Jpn. 12, 1203 (1957).

[12] U. Seifert, Stochastic thermodynamics, fluctuation theorems and molecular machines, Rep. Prog. Phys. 75, 126001 (2012).

[13] C. Jarzynski, Equalities and inequalities: Irreversibility and the second law of thermodynamics at the nanoscale, Annu. Rev. Condens. Matter Phys. 2, 329 (2011).

[14] U. Seifert, First and Second Law of Thermodynamics at Strong Coupling, Phys. Rev. Lett. 116, 020601 (2016).

[15] P. Strasberg, G. Schaller, N. Lambert, and T. Brandes, Nonequilibrium thermodynamics in the strong coupling and non-Markovian regime based on a reaction coordinate mapping, New J. Phys. 18, 073007 (2016).

[16] P. Strasberg and M. Esposito, Stochastic thermodynamics in the strong coupling regime: An unambiguous approach based on coarse graining, Phys. Rev. E 95, 062101 (2017).

[17] C. Jarzynski, Stochastic and Macroscopic Thermodynamics of Strongly Coupled Systems, Phys. Rev. X 7, 011008 (2017).

[18] P. Talkner and P. Hänggi, Colloquium: Statistical mechanics and thermodynamics at strong coupling: quantum and classical, arXiv:1911.11660 [Rev. Mod. Phys. (to be published).]

[19] R. Metzler, E. Barkai, and J. Klafter, Anomalous Diffusion and Relaxation Close to Thermal Equilibrium: A Fractional Fokker-Planck Equation Approach, Phys. Rev. Lett. 82, 3563 (1999).

[20] R. Metzler and J. Klafter, The random walk's guide to anomalous diffusion: a fractional dynamics approach, Phys. Rep. 339, 1 (2000).

[21] J.-H. Jeon and R. Metzler, Inequivalence of time and ensemble averages in ergodic systems: Exponential versus power-law relaxation in confinement, Phys. Rev. E 85, 021147 (2012).

[22] I. M. Sokolov and J. Klafter, From diffusion to anomalous diffusion: A century after Einstein's Brownian motion, Chaos 15, 026103 (2005).

[23] M. Baiesi and C. Maes, An update on the nonequilibrium linear response, New J. Phys. 15, 013004 (2013).

[24] C. Maes, Frenetic Bounds on the Entropy Production, Phys. Rev. Lett. 119, 160601 (2017).

[25] V. Blickle, T. Speck, L. Helden, U. Seifert, and C. Bechinger, Thermodynamics of a Colloidal Particle in a 
Time-Dependent Nonharmonic Potential, Phys. Rev. Lett. 96, 070603 (2006).

[26] T. M. Hoang, R. Pan, J. Ahn, J. Bang, H. Quan, and T. Li, Experimental Test of the Differential Fluctuation Theorem and a Generalized Jarzynski Equality for Arbitrary Initial States, Phys. Rev. Lett. 120, 080602 (2018).

[27] J.-H. Jeon, N. Leijnse, L. B. Oddershede, and R. Metzler, Anomalous diffusion and power-law relaxation of the time averaged mean squared displacement in worm-like micellar solutions, New J. Phys. 15, 045011 (2013).

[28] D. Collin, F. Ritort, C. Jarzynski, S. B. Smith, I. Tinoco, and C. Bustamante, Verification of the Crooks fluctuation theorem and recovery of RNA folding free energies, Nature (London) 437, 231 (2005).

[29] E. Dieterich, J. Camunas-Soler, M. Ribezzi-Crivellari, U. Seifert, and F. Ritort, Single-molecule measurement of the effective temperature in non-equilibrium steady states, Nat. Phys. 11, 971 (2015).

[30] J. Camunas-Soler, A. Alemany, and F. Ritort, Experimental measurement of binding energy, selectivity, and allostery using fluctuation theorems, Science 355, 412 (2017).

[31] K. Hayashi, H. Ueno, R. Iino, and H. Noji, Fluctuation Theorem Applied toF1-ATPase, Phys. Rev. Lett. 104, 218103 (2010).

[32] T. Hatano and S.-i. Sasa, Steady-State Thermodynamics of Langevin Systems, Phys. Rev. Lett. 86, 3463 (2001).

[33] C. Maes, K. Netočný, and B. Wynants, Monotonic Return to Steady Nonequilibrium, Phys. Rev. Lett. 107, 010601 (2011).

[34] M. Polettini and M. Esposito, Nonconvexity of the relative entropy for Markov dynamics, Phys. Rev. E 88, 012112 (2013).

[35] C. Maes, Frenesy: Time-symmetric dynamical activity in nonequilibria, Phys. Rep. 850, 1 (2020).

[36] T. Speck and U. Seifert, Integral fluctuation theorem for the housekeeping heat, J. Phys. A 38, L581 (2005).

[37] R. Chétrite, S. Gupta, I. Neri, and É. Roldán, Martingale theory for housekeeping heat, Europhys. Lett. 124, 60006 (2019).

[38] H.-M. Chun and J.D. Noh, Universal property of the housekeeping entropy production, Phys. Rev. E 99, 012136 (2019).

[39] Z. Lu and O. Raz, Nonequilibrium thermodynamics of the Markovian Mpemba effect and its inverse, Proc. Natl. Acad. Sci. U.S.A. 114, 5083 (2017).

[40] I. Klich, O. Raz, O. Hirschberg, and M. Vucelja, Mpemba Index and Anomalous Relaxation, Phys. Rev. X 9, 021060 (2019).

[41] N. Shiraishi and K. Saito, Information-Theoretical Bound of the Irreversibility in Thermal Relaxation Processes, Phys. Rev. Lett. 123, 110603 (2019).

[42] D. Hartich and A. Godec, Duality between relaxation and first passage in reversible Markov dynamics: rugged energy landscapes disentangled, New J. Phys. 20, 112002 (2018).

[43] D. Hartich and A. Godec, Interlacing relaxation and firstpassage phenomena in reversible discrete and continuous space Markovian dynamics, J. Stat. Mech. (2019) 024002.

[44] I. A. Martínez, E. Roldán, L. Dinis, D. Petrov, J. M. R. Parrondo, and R. A. Rica, Brownian Carnot engine, Nat. Phys. 12, 67 (2016).
[45] I. A. Martínez, E. Roldán, J. M. R. Parrondo, and D. Petrov, Effective heating to several thousand kelvins of an optically trapped sphere in a liquid, Phys. Rev. E 87, 032159 (2013).

[46] S. de Lorenzo, M. Ribezzi-Crivellari, J. Arias-Gonzalez, S. Smith, and F. Ritort, A temperature-jump optical trap for single-molecule manipulation, Biophys. J. 108, 2854 (2015).

[47] J. Gladrow, M. Ribezzi-Crivellari, F. Ritort, and U. F. Keyser, Experimental evidence of symmetry breaking of transition-path times, Nat. Commun. 10, 55 (2019).

[48] G. Sitters, N. Laurens, E. de Rijk, H. Kress, E. Peterman, and G. Wuite, Optical pushing: A tool for parallelized biomolecule manipulation, Biophys. J. 110, 44 (2016).

[49] S. Kullback and R. Leibler, On information and sufficiency, Ann. Math. Stat. 22, 79 (1951).

[50] J. L. Lebowitz and P. G. Bergmann, Irreversible gibbsian ensembles, Ann. Phys. (N.Y.) 1, 1 (1957).

[51] M. C. Mackey, The dynamic origin of increasing entropy, Rev. Mod. Phys. 61, 981 (1989).

[52] H. Qian, A decomposition of irreversible diffusion processes without detailed balance, J. Math. Phys. 54, 053302 (2013).

[53] C. Van den Broeck and M. Esposito, Three faces of the second law. II. Fokker-Planck formulation, Phys. Rev. E 82, 011144 (2010).

[54] M. Esposito and C. Van den Broeck, Three Detailed Fluctuation Theorems, Phys. Rev. Lett. 104, 090601 (2010).

[55] S. Vaikuntanathan and C. Jarzynski, Dissipation and lag in irreversible processes, Europhys. Lett. 87, 60005 (2009).

[56] M. Doi and S.F. Edwards, The Theory of Polymer Dynamics (Clarendon Press, Oxford, 1988).

[57] $T>T_{\text {eq }}$ implies $\tilde{T}>1$ and $T<T_{\text {eq }}$ implies $0<\tilde{T}<1$.

[58] The name comes from the fact that $\mathcal{U}(\mathbf{q})$ delivers the mean force, i.e., $-\nabla_{\mathbf{q}} \mathcal{U}(\mathbf{q})=-\left\langle\nabla_{\mathbf{x}} U(\mathbf{x}) \delta[\mathbf{\Gamma}(\mathbf{x})-\mathbf{q}]\right\rangle$.

[59] J.G. Kirkwood, Statistical mechanics of fluid mixtures, J. Chem. Phys. 3, 300 (1935).

[60] See Supplemental Material at http://link.aps.org/ supplemental/10.1103/PhysRevLett.125.110602 for details of calculations, proofs, and supplementary examples, as well as Refs. [61-72].

[61] I. Chatzigeorgiou, Bounds on the lambert function and their application to the outage analysis of user cooperation, IEEE Commun. Lett. 17, 1505 (2013).

[62] A. Godec, T. Ukmar, M. Gaberšček, and F. Merzel, Inversion of pore size dependence of solute transport kinetics from increasingly attractive ordered porous matrix, Europhys. Lett. 92, 60011 (2010).

[63] E. B. Mpemba and D. G. Osborne, Cool?, Phys. Educ. 14, 410 (1979).

[64] M. Jeng, The Mpemba effect: When can hot water freeze faster than cold?, Am. J. Phys. 74, 514 (2006).

[65] J. I. Katz, When hot water freezes before cold, Am. J. Phys. 77, 27 (2009).

[66] P. Chaddah, S. Dash, K. Kumar, and A. Banerjee, Overtaking while approaching equilibrium, arXiv:1011.3598.

[67] P. A. Greaney, G. Lani, G. Cicero, and J. C. Grossman, Mpemba-like behavior in carbon nanotube resonators, Metall. Mater Trans. A 42, 3907 (2011). 
[68] C. Hu, J. Li, S. Huang, H. Li, C. Luo, J. Chen, S. Jiang, and L. An, Conformation directed mpemba effect on polylactide crystallization, Cryst. Growth Des. 18, 5757 (2018).

[69] Y.-H. Ahn, H. Kang, D.-Y. Koh, and H. Lee, Experimental verifications of $\{$ Mpemba\}-like behaviors of clathrate hydrates, Korean J. Chem. Eng. 33, 1903 (2016).

[70] A. Gijón, A. Lasanta, and E. R. Hernández, Paths towards equilibrium in molecular systems: The case of water, Phys. Rev. E 100, 032103 (2019).

[71] M. Baity-Jesi et al. (J. Collaboration), The Mpemba effect in spin glasses is a persistent memory effect, Proc. Natl. Acad. Sci. U.S.A. 116, 15350 (2019).

[72] I. Klich and M. Vucelja, Solution of the Metropolis dynamics on a complete graph with application to the Markov chain Mpemba effect, arXiv:1812.11962.
[73] S. Yang, J. B. Witkoskie, and J. Cao, Single-molecule dynamics of semiflexible Gaussian chains, J. Chem. Phys. 117, 11010 (2002).

[74] C. Joo, H. Balci, Y. Ishitsuka, C. Buranachai, and T. Ha, Advances in single-molecule fluorescence methods for molecular biology, Annu. Rev. Biochem. 77, 51 (2008).

[75] G. J. Moro, Kinetic equations for site populations from the Fokker-Planck equation, J. Chem. Phys. 103, 7514 (1995).

[76] T. Schmiedl and U. Seifert, Efficiency at maximum power: An analytically solvable model for stochastic heat engines, Europhys. Lett. 81, 20003 (2008).

[77] H. Ouerdane, Y. Apertet, C. Goupil, and P. Lecoeur, Continuity and boundary conditions in thermodynamics: From Carnot's efficiency to efficiencies at maximum power, Eur. Phys. J. Special Topics 224, 839 (2015). 